\title{
Simulation of nonorganic scintillation detector response for the problems of active interrogation by tagged neutron technology
}

\author{
V. F. Batyaev, S. G. Belichenko \& R. R. Bestaev \\ Dukhov All-Russia Research Institute of Automatics (VNIIA), Russia
}

\begin{abstract}
The paper concerns potential of numerical simulation for assessment of characteristics of tagged neutron device. The CERN Geant4 code and TPT2 (Toolkit for Particle Transport) code developed in VNIIA were used to simulate the $\gamma$-neutron transport in components of the tagged neutron device and recording of ionizing radiation by the scintillation detector. The numerical simulation was corrected and validated by experimentations with the model of the device of the same geometry, type of detector, materials. At first, we obtained a spectral response of LYSO scintillator under $\gamma$ - and neutron radiation and satisfied that it was close to the measured data. Using the detector response, the instrumental spectra of the tagged neutron device including the neutron generator, LYSO scintillating detector and tetryl/sugar as an object of interrogation were calculated. A good agreement of coefficients of decomposition of experimental and calculated spectra let us validate that Geant 4 and TPT2 codes can be used for the reliable numerical simulation of various configurations of the tagged neutron device.

Keywords: explosive detection, fast neutrons, tagged neutrons, associated particle imaging, Monte-Carlo simulation.
\end{abstract}

\section{Introduction}

Effective technologies for inspection of baggage and cargo containers are in a great demand to prevent illicit trafficking of explosives. Inspection systems based on tagged neutron (TN) technology $[1,2]$ are actively developed in some laboratories worldwide. In opposite to X-ray scanners, the TN technology allows measuring the chemical composition of hidden substances that highly enhance their identification. 
An idea of TN technology is as follows. A neutron generator emits $14 \mathrm{MeV}$ neutrons in $\mathrm{D}(\mathrm{T}, \mathrm{n}) \alpha$ reaction. An associated $\alpha$-particle (emitted simultaneously in the opposite direction to a neutron) is recorded by a position sensitive detector. The direction of the neutron escape is deduced from the coordinates of recorded $\alpha$-particle. The tagged neutron can induce gamma-rays with the characteristic spectral lines in the inspected object. The location of nucleon emitted the gammaquantum can be derived by TOF (time of flight - time between recording of associated alpha-particle and gamma-quantum). The tagged neutron generators of ING-27 [3] type are serially produced by VNIIA along with the wide nomenclature of portable neutron generators.

Configuration of inspection device depends on the number and type of $\gamma$ detectors, type and dimensions of shadow shielding, locations of generator and detectors. Such parameters can be indicated as primary ones as far as they are set at the development stage. The duration of inspection, rate of detection and false alarm, minimal detectable mass are target specifications that are chosen by a user. The inspection system should meet the given target specification; for known dependences between primary parameters and target specification the primary parameters can be optimized by some goal function, e.g. a cost of the system. Along with experimental measurements a computer simulation can provide fast and effective estimation of parameters and their dependencies for various configurations of TN device.

This work is a supplement to a study of these dependences. Some dependencies between the type of detector, type of substance and system target specification are represented in [4].

\section{Measurements}

The measured energy spectra of $\gamma$ rays induced by tagged neutrons provide a primary information on the object. The measurements were implemented at the experimental setup (Fig. 1) including ING-27 [5] neutron generator with build-in 9 pixel alpha-detector and 3" x 3" LYSO (Prelude 420, Saint-Gobain) gammadetector. The tungsten shadow shielding between the generator and detector

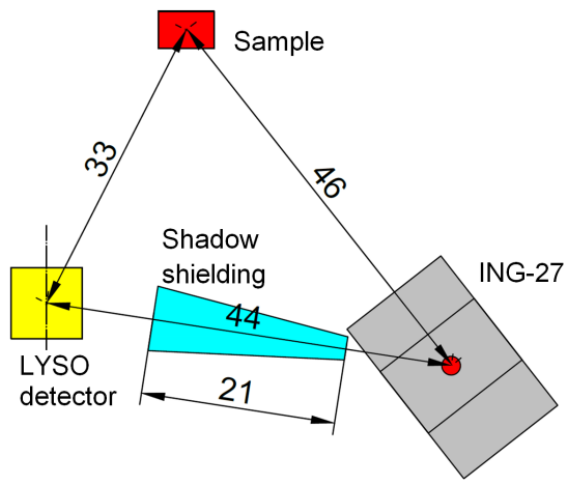

Figure 1: Geometry of experimental setup, dimensions in $\mathrm{cm}$. 
reduces random coincidence background. An intensity of ING-27 was $5 \cdot 10^{7}$ neutron/s. Associated alpha-particles were recorded by single pixel alpha detector that tagged $0.2 \%$ of all emitted neutrons. Configuration of experiment and sample dimensions were chosen in such a way that a sample was completely contained in a voxel (minimal volume resolvable by $\mathrm{TN}$ technology). The examined samples are listed in Table 1.

Table 1: Examined samples.

\begin{tabular}{|l|l|c|}
\hline Composition & Sample parameters & $\begin{array}{c}\text { Dimensions } \\
(\mathrm{cm})\end{array}$ \\
\hline $\mathrm{CH}_{2}$ & Polyethylene, $\mathrm{m}=0.3 \mathrm{~kg}, \rho=0.94 \mathrm{~g} / \mathrm{cm}^{3}$ & $8 \times 8 \times 5$ \\
\hline $\mathrm{H}_{2} \mathrm{O}$ & Water, $\mathrm{m}=0.5 \mathrm{~kg}, \rho=1.0 \mathrm{~g} / \mathrm{cm}^{3}$ & 8 (cube) \\
\hline $\mathrm{C}_{3} \mathrm{H}_{6} \mathrm{~N}_{6}$ & Melamine, $\mathrm{m}=0.55 \mathrm{~kg}, \rho=1.074 \mathrm{~g} / \mathrm{cm}^{3}$ & 8 (cube) \\
\hline $\mathrm{C}_{7} \mathrm{~N}_{7.5} \mathrm{~N}_{5} \mathrm{O}_{7.8}$ & Tetryl imitator, $\mathrm{m}=0.3 \mathrm{~kg}, \rho=1.544 \mathrm{~g} / \mathrm{cm}^{3}$ & $\varnothing 9 \times 3$ \\
\hline $\mathrm{H}_{12} \mathrm{C}_{6} \mathrm{O}_{6}$ & Sugar, $\mathrm{m}=0.5 \mathrm{~kg}, \rho=0.98 \mathrm{~g} / \mathrm{cm}^{3}$ & 8 (cube) \\
\hline
\end{tabular}

Polyethylene, water and melamine samples were interrogated during 4 hours each, and tetryl imitator and sugar -2 hours. The outcome information from experiment is the differential (energy and time) response spectra, Fig. 2.

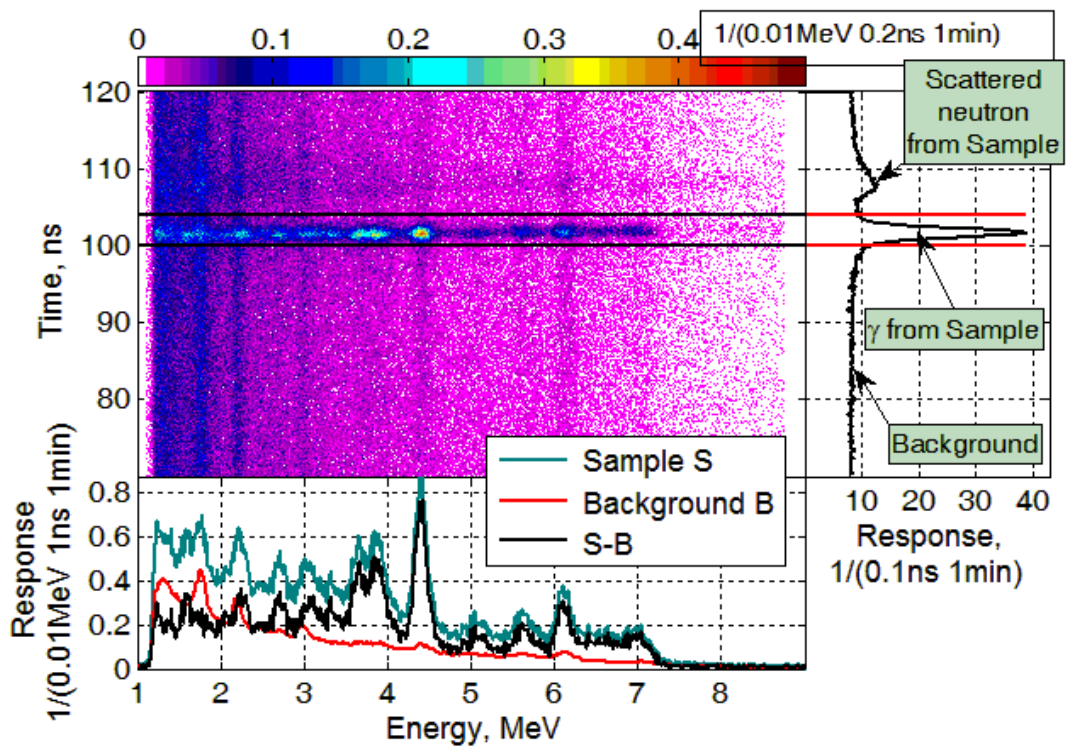

Figure 2: TOF (time of flight) and energy spectra for tetryl imitator.

TOF (time of flight) and energy spectra are given in Fig. 2. The key information for elemental analysis is the energy spectra. 


\section{Simulation of spectral response LYSO detector under $\gamma$ and neutron radiation}

The CERN Geant4 [6] code and TPT2 (Toolkit for Particle Transport) [7] code developed in VNIIA were used to simulate the $\gamma$-neutron transport in objects of a model of experimental setup and recording of ionizing radiation by the LYSO scintillator. A spectral response was represented by the MR matrix. The (Ei,Ej) elements of the MR matrix are the detector response in channel Ei as a result of impact of particle with energy $\mathrm{Ej}$ on the scintillator. The isotropic source of ionizing particles was located at $33 \mathrm{~cm}$ from the detector at the $45^{\circ}$ angle to the scintillator axis. Such geometry made it possible to account a variable range of incoming particles. The energy of incoming $\gamma$-rays was varied from $0.5 \mathrm{MeV}$ to $9 \mathrm{MeV}$ with a $0.01 \mathrm{MeV}$ step.

In case of neutron impact, a nonlinearity between specific ionization $\mathrm{dE} / \mathrm{dx}$ and specific scintillation $\mathrm{dL} / \mathrm{dx}$ for heavy nucleus initiated by neutrons was corrected by Birks scaling [8]:

$$
\frac{d L}{d x} \sim \frac{1}{1+k B \frac{d E}{d x}}
$$

According to [9], $\mathrm{kB}=7.6(\mathrm{MeV} / \mu \mathrm{m})^{-1}$ for LYSO scintillator. The energy of neutrons was varied from $10^{-11} \mathrm{MeV}$ to $14 \mathrm{MeV}$ with a logarithmic step, 552 energy bins in total.

Results of the spectral response simulation of the LYSO scintillator are given in Fig. 3. It can be seen that the response substantially falls down for the neutron energy below $1 \mathrm{eV}$.
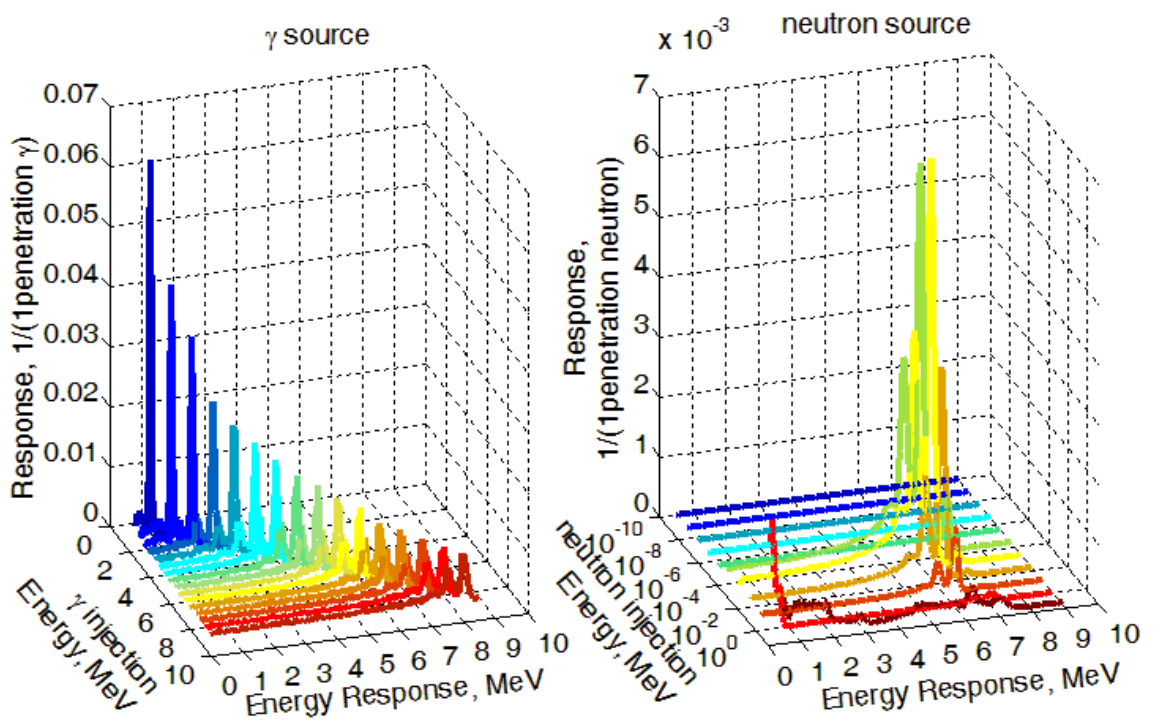

Figure 3:

Spectral response matrix for LYSO detector. 
Results of such simulations were normalized on a flux of particles penetrating the scintillator body. Such normalization allows recovering the detector spectral response for known spectral flux $\mathbf{f}(\mathrm{Ei})$ of incident particles by mathematical convolution:

$$
\mathbf{R}(\mathbf{E i})=\operatorname{MR}(\mathbf{E i}, \mathbf{E j})^{*} \mathbf{f}(\mathbf{E j})^{T}
$$

\section{Measurements vs simulations}

\subsection{Energy spectra}

The experimental and calculated energy spectra of $\gamma$-rays induced by tagged neutrons in the samples are compared in Fig. 4. The geometry of the model corresponds to the drawing in Fig. 1, but without a shadow shielding.

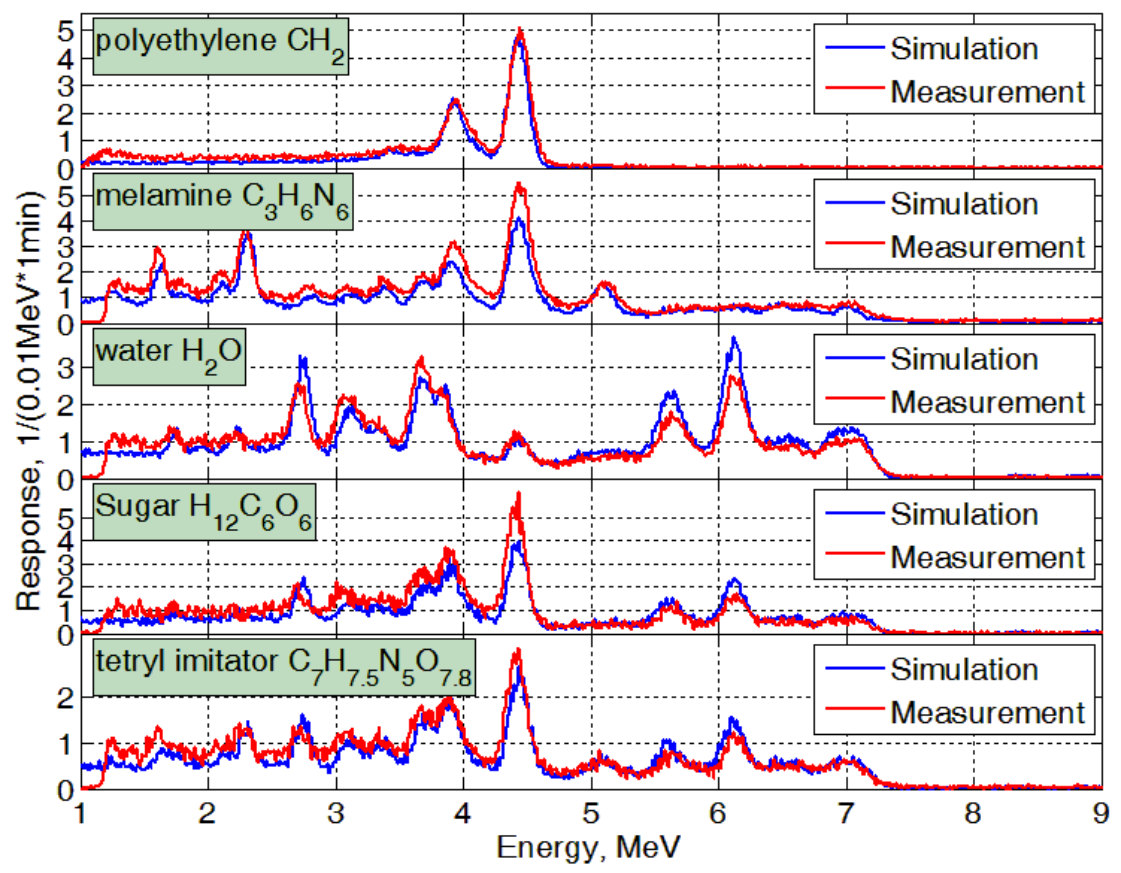

Figure 4: Experimental and calculated energy spectra of $\gamma$-rays from samples recorded by the LYSO detector.

Fig. 5 represents the detector spectral responses from scattered neutrons.

Simulated and experimental spectra are close by spectral density and characteristic lines. It confirms a good agreement between measurements and simulation using the algorithm of convolution upon spectrum of incident particles. Some disagreement for neutrons in the range of $1-4.5 \mathrm{MeV}$ needs detailed examination of simulation algorithms in case of neutron bombardment. 


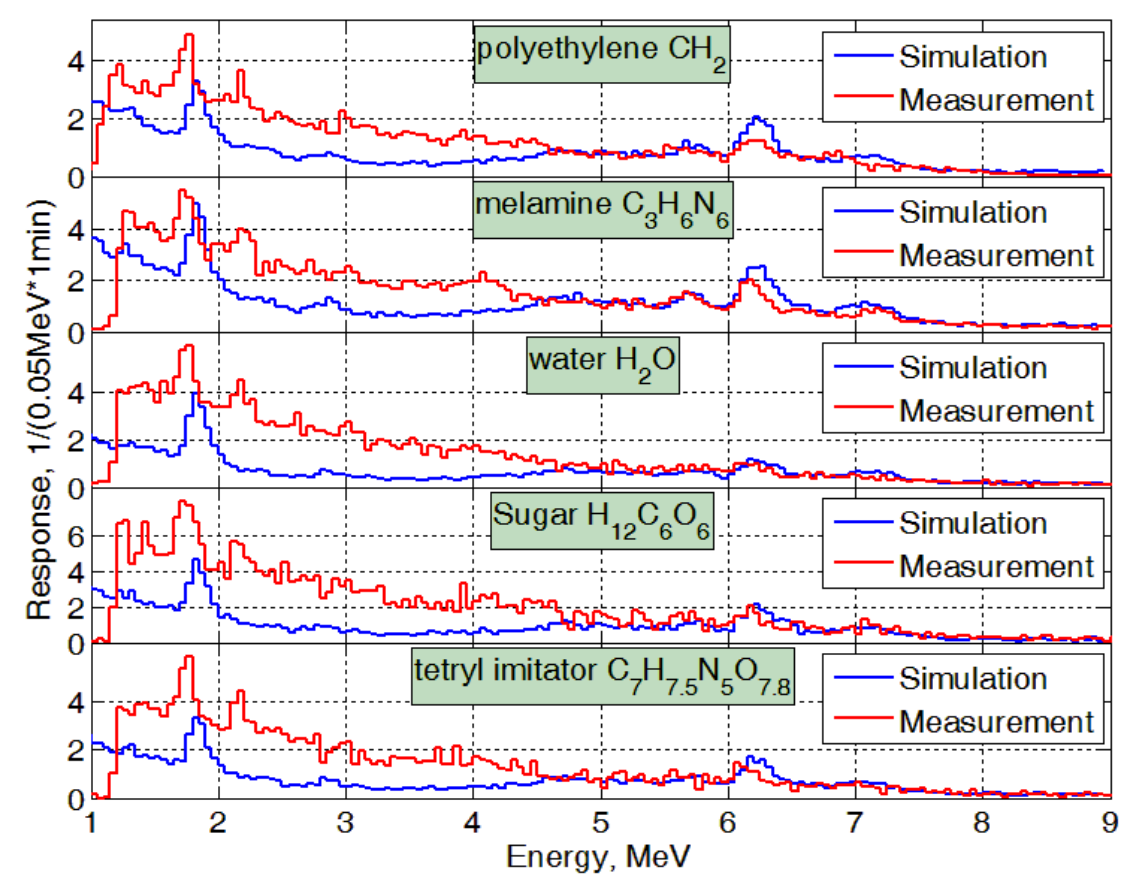

Figure 5: Energy spectrum of LYSO detector under scattered neutron radiation from samples.

\subsection{Decomposition coefficients}

For additional validation of simulation model of the experimental TN device one can compare a distribution of decomposition coefficients of the spectrum $F$ from the sample. These coefficients are the solution of the system of liner algebraic equations:

$$
\mathrm{A} * \mathrm{X}=\mathrm{F}
$$

where $\mathrm{A}$ is a matrix representing the pure (elemental) spectra of C, N, O that could be obtained by simulation or measurement of spectra from the $\mathrm{CH}_{2}, \mathrm{C}_{3} \mathrm{H}_{6} \mathrm{~N}_{6}, \mathrm{H}_{2} \mathrm{O}$ samples; $\mathrm{X}$ is a molar ratio of elements $\mathrm{C}, \mathrm{N}, \mathrm{O}$ in the interrogated substance (tetryl imitator/sugar). Coefficients normalized by equations

$$
x=\frac{\sqrt{2} \cdot(X(1)+0.5 \cdot X(2))}{X(1)+X(2)+X(3)} \quad y=\frac{\sqrt{6} \cdot 0.5 \cdot X(2)}{X(1)+X(2)+X(3)}
$$

represent the decomposition coefficients in CNO triangle (Fig. 6 [4]). Dispersion ellipses cover $95 \%$ of decomposition coefficients of substances. A quadratic value of confidence region

$$
S^{2}=2 \pi \sigma_{x} \sigma_{y} \sqrt{1-\rho_{x y}}
$$

is an important parameter of the distribution. Estimated $\mathrm{S}^{2}$ values of $68 \%$ region are listed in Table 2. 

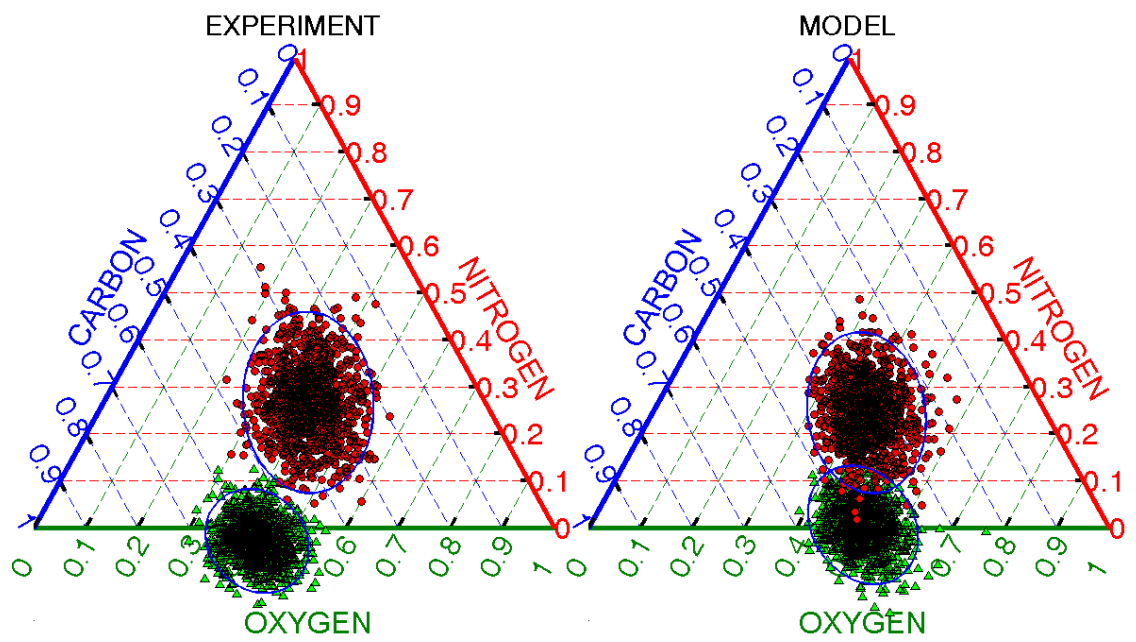

Figure 6: Distribution of decomposition coefficients, obtained by $\mathrm{TN}$ technology for sugar $\Delta$ and tetryl imitator o.

Table 2: Estimated S2 values of $68 \%$ region.

\begin{tabular}{|l|c|c|}
\hline & Tetryl imitator & Sugar \\
\hline Measurement, $\mathrm{S}^{2}$ & $0.044(0.0400 .050)$ & $0.020(0.0180 .022)$ \\
\hline Simulation, $\mathrm{S}^{2}$ & $0.036(0.0330 .039)$ & $0.024(0.0220 .026)$ \\
\hline
\end{tabular}

A comparison of experimental and calculated distributions (Fig. 6 and Table 2) demonstrates their good correspondence. A slight shift of mean of experimental distribution for sugar from theoretical one $\left(\mathrm{C}=0.5, \mathrm{O}=0.5 \mathrm{H}_{12} \mathrm{C}_{6} \mathrm{O}_{6}\right)$ can be caused by underestimation of impurities in sugar.

\section{Conclusion}

The main goal of this work was a validation of numerical simulation method for assessment of parameters of $\mathrm{TN}$ devices by comparison of experimental and calculated gamma-detector spectra from several samples at the given configuration of the device. The Geant 4 and TPT 2 codes were used for calculation of spectral response of LYSO 3"x 3" gamma-detector under gamma-neutron radiation and spectra of gamma-rays induced by tagged neutrons. A calculated matrix of spectral response of the detector provided convenient computation of energy spectra of detector upon incident gamma-neutron radiation. In the nearest future we are planning to calculate spectral response matrixes for $\mathrm{BGO}$, $\mathrm{LaBr}_{3}(\mathrm{Ce}), \mathrm{NaI}$ detectors. 
A correspondence between calculated and measured spectra let us conclude that the numerical simulation can be used for reliable estimation of key parameters of inspection systems at various configurations and optimization of these parameters by the given goal function (e.g., cost of the inspection system).

\section{References}

[1] B. Perot et al., Development of the EURITRACK tagged neutron inspection system. Nucl. Instr. and Meth. In Phys. Res. B 261 (2007) pp. 295-298.

[2] Robert C. Runkle et al., Photon and neutron interrogation techniques for chemical explosives detection in air cargo: A critical review. Nucl. Instr. and Meth. In Phys. Res. A 603 (2009) pp. 510-528.

[3] Kh.E. Bagdasaryan, et al., Parameters of explosives detection through tagged neutron method. Nucl. Instr. and Meth. In Phys. Res. A (2015), http://dx.doi.org/10.1016/j.nima.2014.11.111.

[4] V.F. Batyaev et al., Int. J. Mod. Phys. Conf. Ser. 27, 1460131 (2014), www.worldscientific.com/doi/abs/10.1142/S2010194514601318.

[5] ING-27 gas-filled neutron tube based neutron generator http://www.vniia.ru/eng/ng/element.html.

[6] Geant4 Collaboration. Geant4 User's Guide for Application Developers. http://geant4.web.cern.ch/geant4/UserDocumentation/UsersGuides/For ApplicationDeveloper/fo/BookForAppliDev.pdf.

[7] CFAR, TPT (Toolkit for Particle Transport). http://www.vniia.ru/cfpi /eng/wptpt.html.

[8] В.О. Вяземский, и др., Сцинтилляционный метод в радиометрии, Гос. Изд-во в области атомной науки и техники, М. - 1961.

[9] Y. KOBA, et al., Scintillation Efficiency of Inorganic Scintillators for Intermediate-Energy Charged Particles. Progress in Nuclear Science and Technology, Vol. 1, pp. 218-221 (2011). 